\title{
Quantum Graphs Whose Spectra Mimic the Zeros of the Riemann Zeta Function
}

\author{
Jack Kuipers, ${ }^{*}$ Quirin Hummel, ${ }^{\dagger}$ and Klaus Richter \\ Institut für Theoretische Physik, Universität Regensburg, D-93040 Regensburg, Germany
}

(Received 18 July 2013; published 21 February 2014)

\begin{abstract}
One of the most famous problems in mathematics is the Riemann hypothesis: that the nontrivial zeros of the Riemann zeta function lie on a line in the complex plane. One way to prove the hypothesis would be to identify the zeros as eigenvalues of a Hermitian operator, many of whose properties can be derived through the analogy to quantum chaos. Using this, we construct a set of quantum graphs that have the same oscillating part of the density of states as the Riemann zeros, offering an explanation of the overall minus sign. The smooth part is completely different, and hence also the spectrum, but the graphs pick out the low-lying zeros.
\end{abstract}

DOI: 10.1103/PhysRevLett.112.070406

PACS numbers: 03.65.Sq, 02.10.De, 05.45.Mt

The Riemann zeta function $\zeta(s)$ encodes the distribution of the prime numbers and therefore plays a central role in number theory. It is the analytic continuation of the infinite sum over integers $\sum_{n=1}^{\infty} n^{-s}$ to $\operatorname{Re} s \leq 1$. In fact its zeros can be used to obtain the prime counting function with Heaviside steps at each prime. The famous Riemann hypothesis states that all the zeros, aside from the trivial zeros at the negative even numbers, lie on a critical line with real part $1 / 2$. Writing the nontrivial zeros, which come in complex conjugate pairs, as $s_{n}=1 / 2 \pm \mathrm{i} t_{n}$, the $t_{n}$ would all be real if the Riemann hypothesis is true. Proving the hypothesis remains one of the outstanding problems in mathematics, and would also prove the many propositions based on it.

Despite its number-theoretical background, the Riemann zeta function appears in the study of a range of different physical systems [1] including recently the freezing transition in random energy landscapes [2,3]. In a connection dating to Montgomery [4] and Dyson, the zeros are particularly strongly related to the eigenvalues of random matrices from the Gaussian unitary ensemble. Along with numerical evidence that they share the same statistics [5], random matrices have also been used to obtain conjectures about the moments of $\zeta(s)$ [6].

Already Hilbert and Pólya recognized (as discussed in [4]) that if the $t_{n}$ could be identified as the eigenvalues of a Hermitian operator, they would necessarily be real and the hypothesis proved. This has triggered the search for a quantum system described by such a Hermitian Hamiltonian. Moreover, the fact that the spectra of quantum systems with chaotic classical counterparts obey random matrix statistics [7], like the Riemann zeros, suggests seeking an appropriate quantum chaotic system. Further hints [8] about the nature of such a system derive from an analogy starting from the density of states. Placing a delta function at each zero along the critical line, the density of states $d(t)=\sum_{n=1}^{\infty} \delta\left(t-t_{n}\right)=\bar{d}(t)+d^{\text {osc }}(t)$ can be considered as consisting of two parts: a smooth average background $\bar{d}(t)$ overlaid with an oscillating part $d^{\text {osc }}(t)$. For the zeros of the Riemann zeta function the smooth part (see, e.g., [8])

$$
\bar{d}(t)=\frac{1}{2 \pi} \ln \frac{t}{2 \pi}+O\left(\frac{1}{t^{2}}\right)
$$

is logarithmically increasing while the oscillating part has the divergent expression $[8,9]$

$$
d^{\mathrm{osc}}(t)=-\frac{1}{\pi} \sum_{p} \sum_{m=1}^{\infty} \frac{\ln p}{p^{m / 2}} \cos (\operatorname{tm} \ln p),
$$

involving a sum over all primes $p$ and their "repetitions" $m$. One can enforce convergence by smoothing with a Gaussian of width $\epsilon$, washing out terms with $\epsilon m \ln (p) \gg 2 \pi$. Plotting the smoothed sum of the remaining primes in Fig. 1 we see peaks exactly at the Riemann zeta zeros along with the overall divergence at $t=0$. As $t$ increases, the zeros come closer according to (1), so with fixed $\epsilon$ we eventually stop being able to resolve them. The mean part of the density of states can also be seen in Fig. 1 as the lower curve needed to map the gap between the peaks to the axis. See the Supplemental Material [10] for the density in the complex plane.

The formula in (2) is remarkably similar to Gutzwiller's trace formula [11] for the oscillating part of the density of energy states of chaotic quantum systems

$$
d^{\mathrm{osc}}(E) \approx \frac{1}{\pi \hbar} \sum_{\gamma} \sum_{m=1}^{\infty} T_{\gamma} A_{\gamma, m} \cos \left(\frac{m S_{\gamma}}{\hbar}-\frac{m \pi \mu_{\gamma}}{2}\right)
$$

The sum is over the classical primitive periodic orbits $\gamma$ of the system and their repetitions $m$. Each orbit has period $T_{\gamma}$, stability amplitude $A_{\gamma}$, reduced action $S_{\gamma}$ and Maslov index $\mu_{\gamma}$. The close correspondence between (3) and (2) becomes 


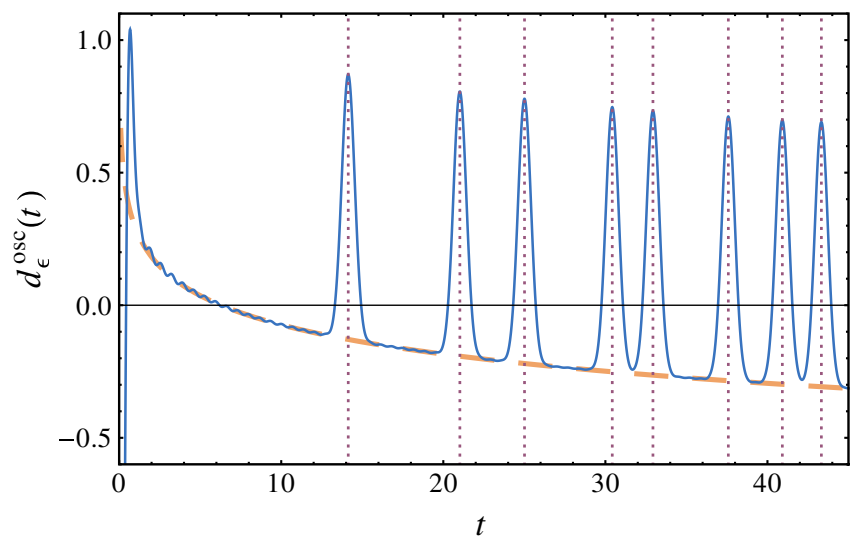

FIG. 1 (color online). Solid blue: oscillating part (2) of the density of zeros of the Riemann zeta function smoothed with a Gaussian of width $\epsilon=0.4$. The sums over $p$ and $m$ are truncated to $\mathrm{em} \ln p \leq 3.7$. Dotted purple: exact zeros of the Riemann zeta function. Dashed orange: negative of smooth part (1).

evident if we associate primes $p$ in (2) with primitive periodic orbits $\gamma$ in (3).

For a quantum chaotic system to match the Riemann zeta function, a wide range of properties can be deduced from this analogy including that the dynamics should be quasi one dimensional and without time reversal symmetry [8]. Berry and Keating realized that many of the properties are satisfied by the simple Hamiltonian $H=x p$, except that the motion is unbounded [8]. With a truncation near the origin, the semiclassical mean density of states is also the same as (1) but the problem was in finding boundary conditions to give a Hermitian operator with real eigenvalues. Various related operators have since been obtained which keep the same mean part of the density of states as the Riemann zeros [12-16]. However these extensions still miss two of the trickiest properties that the quantum system should have and their spectra do not match the Riemann zeros.

A closer comparison of (3) to (2) highlights these two properties. Namely that the periodic orbits should (i) have primitive lengths $\ln (p)$ corresponding to all primes $p$ and (ii) all have a Maslov phase of $\pi$ to obtain the minus sign in (2). The Maslov phase is the term $m \pi \mu_{\gamma} / 2$ in (3) so the second requirement cannot hold for all $m$. Overcoming this contradiction is the aim of this paper.

To enforce that the periodic orbits only have particular lengths of $\ln (p)$ we turn to quantum graphs where the Schrödinger equation acts on the bonds of a network. Quantum graphs arise as simplified models in a range of physical applications [17], providing for example the spectra and dispersion relations of carbon nano-structures like graphene [18], and fittingly are used to capture the essence of the quantum behavior of chaotic systems $[19,20]$. The bonds are one dimensional so we directly satisfy one of the properties needed while we later tune the lengths and connections between the bonds to obtain the two requirements listed above. Focusing on the oscillating part of the density of states, we then construct infinite graphs which match (2). Since the zeros of the Riemann zeta function come in complex conjugate pairs with $\pm t_{n}$, they behave more like a wave number than energy spectrum [used in the analogy with (3)]. We therefore now treat $t_{n}$ as wave numbers $k$, especially since the trace formula for quantum graphs involves $k$ directly. This does not affect the analogy since the $k$, as square roots of positive real energies, correspond to a Hermitian operator.

Quantum graphs.-The density of states for a quantum graph has a mean part $\bar{d}(k)=L_{\text {tot }} / \pi$ given by the total length $L_{\mathrm{tot}}$ of the bonds, counting directed bonds with a factor of $1 / 2$. This is independent of $k$, unlike for the Riemann zeta zeros in (1), but the oscillating part of the density of states [20] reduces to

$$
d^{\mathrm{osc}}(k)=+\frac{1}{\pi} \sum_{\gamma} \sum_{m=1}^{\infty} L_{\gamma} A_{\gamma}^{m} \cos \left(k m L_{\gamma}\right)
$$

if $\sum_{m L_{\gamma=L}} L_{\gamma} A_{\gamma}^{m} \in \mathbb{R}$ for all possible periodic orbit lengths $L$. The length $L_{\gamma}=\sum_{e \in \gamma} L_{e}$ of the primitive orbit $\gamma$ is the sum of the lengths of the edges involved and $A_{\gamma}^{m}=$ $\prod \sigma_{e_{i+1}, e_{i}}^{m}$ the product of the scattering matrix elements the orbit passes through with $e$ being the edges in $\gamma$.

One can then construct a quantum graph with periodic orbits of length $\ln (p)$ by simply setting the lengths of the bonds to the same value, but this gives two problems: (a) if orbits of length $\ln (p)$ and $\ln (q)$ connect at the same vertex, we can have an orbit of length $\ln (p q)$ which is a composite number; (b) if an orbit corresponding to a prime $p$ has a negative prefactor $A_{\gamma}<0$, its repetitions have prefactors $A_{\gamma}^{m}$ giving the even repetitions the wrong sign.

To avoid problem (a), we can simply only connect orbits whose lengths only involve the same prime $p$. In particular we can consider butterfly graphs made up of two identically long directed bonds which meet at a single vertex. The inset of Fig. 2 shows such a butterfly graph.

Along its directed bonds the wave function admits the solutions $\varphi_{1}\left(x_{1}\right)=c_{1} e^{i k x_{1}}$ and $\varphi_{2}\left(x_{2}\right)=c_{2} e^{i k x_{2}}$, where $x_{i}$ are the coordinates along the bonds starting at the vertex and following the direction of the bond. At the vertex, the wave function has to fulfill boundary conditions

$$
\left(\begin{array}{l}
c_{1} \\
c_{2}
\end{array}\right)=S\left(\begin{array}{l}
c_{1} e^{i k L} \\
c_{2} e^{i k L}
\end{array}\right)=\left(\begin{array}{ll}
\sigma_{11} & \sigma_{12} \\
\sigma_{21} & \sigma_{22}
\end{array}\right)\left(\begin{array}{l}
c_{1} e^{i k L} \\
c_{2} e^{i k L}
\end{array}\right)
$$

defined by a scattering matrix $S$, where $L$ is the length of each bond. Each element $\sigma_{j i}$ is the scattering amplitude for an incoming wave on bond $e_{i}$ to an outgoing wave on bond $e_{j}$. In order to preserve total probability current during the scattering process, $S$ needs to be unitary which makes the Hamiltonian self-adjoint [since condition (5) also implies that the first derivatives of the $\varphi_{j}$ are connected by $S$. 


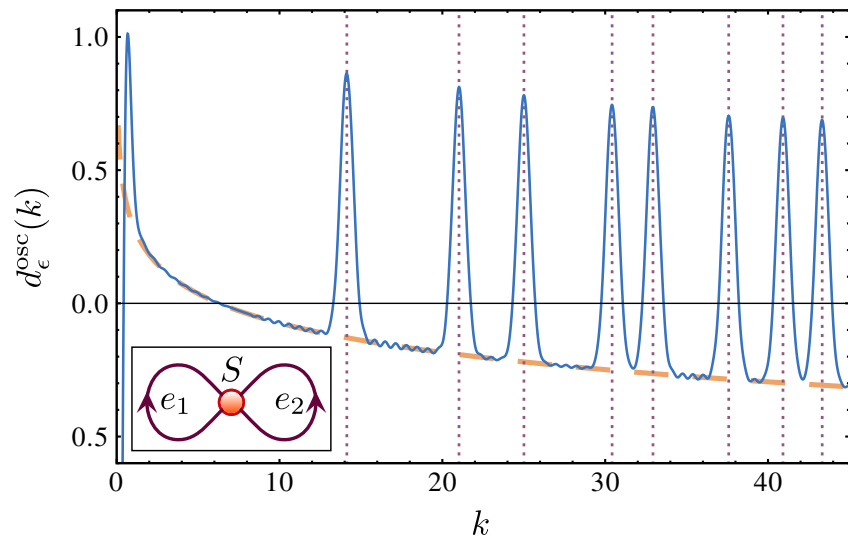

FIG. 2 (color online). Solid blue: density of exact eigenvalues of the set of butterfly graphs (14) smoothed with a Gaussian of width $\epsilon=0.4$ and the mean part subtracted. The set of graphs is truncated at $\epsilon m \ln p \leq 3.7$. Dotted purple: exact zeros of the Riemann zeta function. Dashed orange: negative of the smooth part (1) of the Riemann zeros density. Inset: A butterfly graph. Two directed bonds $e_{1}$ and $e_{2}$ of the same length $L$ meet at a single vertex, characterized by a $2 \times 2$ scattering matrix $S$.

The scattering matrix can therefore be written as

$$
S=U^{\dagger}\left(\begin{array}{cc}
e^{i \theta_{1}} & 0 \\
0 & e^{i \theta_{2}}
\end{array}\right) U
$$

where $U$ is a unitary matrix and $e^{i \theta_{j}}$ are the eigenvalues of $S$. For a given butterfly graph with scattering matrix $S$ and bond length $L$, the wave numbers $k$ which admit wave functions on the graph satisfy $\operatorname{det}\left(1-e^{i k L} S\right)=0$. In terms of the eigenvalues $e^{i \theta_{j}}$ of $S$, this simply means $k=\left(2 \pi z-\theta_{j}\right) / L$ for all integer $z$ so we have a periodic pair of solutions, corresponding to the mean density $L / \pi$. To plot the corresponding spectra of the graphs derived later we subtract the mean part and smooth them with some width $\epsilon$. Terms with $\epsilon L \gg 2 \pi$ can then be excluded from the calculation.

If $\operatorname{Tr} S$ is real we have the further simplification that $\theta_{1}=-\theta_{2}$ (or $\theta_{1}=\theta_{2}+\pi$ for vanishing $\operatorname{Tr} S$ ) and a usefully simple form of the trace formula for butterfly graphs

$$
d^{\mathrm{osc}}(k)=+\frac{1}{\pi} \sum_{m=1}^{\infty} L \operatorname{Tr} S^{m} \cos (k m L) .
$$

Quantum graphs and the sign problem.-The bigger problem is the sign problem of creating orbits with the correct Maslov phase. To overcome this problem we can view the contribution of each prime $p$ as coming from an (infinite) family of graphs that together give the required phase rather than being the result of a single orbit. We now turn to constructing such a set of graphs.

We start by trying to obtain the contribution to the trace formula (2) for a single prime, say $p=2$ and we aim to build a graph that mimics the corresponding term:

$$
-\frac{1}{\pi} \sum_{m=1}^{\infty} \frac{\ln 2}{\sqrt{2}^{m}} \cos (k m \ln 2) .
$$

Comparing the $m=1$ term to that of (7), a butterfly graph with length $L=\ln (2)$ and scattering matrix with $\operatorname{Tr} S=$ $-1 / \sqrt{2}$ would directly give agreement. Labeling the scattering matrix by the subscripts $(p, m)$ for consistency later, we can simply set it to be

$$
S_{2,1}=\left(\begin{array}{cc}
-\frac{1}{2 \sqrt{2}} & \frac{\sqrt{7}}{2 \sqrt{2}} \\
-\frac{\sqrt{7}}{2 \sqrt{2}} & -\frac{1}{2 \sqrt{2}}
\end{array}\right),
$$

though a more general form exists. This butterfly graph then gives a contribution involving $\operatorname{Tr} S_{2,1}^{2}=-3 / 2$ when $m=2$ in (7). This is composed of $1 / 8$ from each second repetition of the bonds of length $\ln (2)$ and $-7 / 4$ from the orbit that covers them both (with twice the primitive length). However, (8) suggests we want a contribution of $-1 / 2$ instead. We can then add a second uncoupled butterfly graph with its own trace formula (7) with two bonds of length $L=\ln (4)$ and a scattering matrix of

$$
S_{2,2}=\left(\begin{array}{cc}
\frac{1}{4} & \frac{\sqrt{15}}{4} \\
-\frac{\sqrt{15}}{4} & \frac{1}{4}
\end{array}\right),
$$

or any unitary matrix with $\operatorname{Tr} S_{2,2}=1 / 2$. As $\ln (4)=$ $2 \ln (2)$, this effectively gives an additional +1 to the $-3 / 2$ contribution from the second power of (9) and therefore the required value.

For $m=3$ and the orbits of length $\ln (8)$, we obtain a contribution of $5 \sqrt{2} / 4$ from $\operatorname{Tr} S_{2,1}^{3}$ while we would want $-\sqrt{2} / 4$. If we add a further uncoupled butterfly graph with two bonds of length $\ln (8)$ and with the same vertex scattering matrix as in (9) they would add an additional $-3 / \sqrt{2}$ to give the required contribution of $m=3$ in (8).

It looks like we could continue this process and define for each prime $p$ a set of independent butterfly graphs ( $p$, $m$ ) each with two bonds of equal length $m \ln (p)$ for all $m \in \mathbb{N}_{+}$. Each pair of bonds is coupled by unitary $2 \times 2$ scattering matrices $S_{p, m}$ with diagonal entries defined so that the whole set conspires to give the sum in (8).

The relations that the butterfly graphs need to satisfy are

$$
\sum_{d \mid m} d \operatorname{Tr} S_{p, d}^{m / d}=-\frac{1}{p^{m / 2}},
$$

involving a sum over divisors of $m$ so that each matrix could be obtained recursively:

$$
\operatorname{Tr} S_{p, m}=-\frac{1}{m p^{m / 2}}-\sum_{d \mid m}^{d<m} \frac{d}{m} \operatorname{Tr} S_{p, d}^{m / d} .
$$


A problem arises however because the sum of divisors of $m$ (divided by $m$ ) are unbounded since

$$
\limsup _{m \rightarrow \infty} \frac{\sum_{d \mid m} \frac{d}{m}}{\log \log m}=e^{\gamma_{\mathrm{e}}},
$$

where $\gamma_{\mathrm{e}}$ is the Euler-Mascheroni constant. Thus the trace of $S_{p, m}$ might need to be larger than 2 in absolute value. For example this happens for $m=24$ for the primes 3 and 5 (among others) allowing no solution of (12). However, we may simply add additional identical copies of the graphs with that length to share the trace between them and find a solution.

Since the traces of all $S_{p, m}$ need to be real [as indicated by (12)], we can replace $S_{p, m}$ by some unitary matrices $U$ and real angles $\pm \theta_{p, m}$ as in (6) (in case of vanishing $\operatorname{Tr} S$ we are free to choose $\theta= \pm \pi / 2$ among others). The spectra of the graphs only depend on the $\theta_{p, m}$ and not on the specific choice of the $U$ so we work with the angles directly. The following prescription then defines the eigenvalues of the whole graph including $l_{p, m}$ copies of the butterfly graph ( $p$, $m$ ) recursively:

$$
T_{p, m} \equiv \frac{1}{2 m p^{m / 2}}+\sum_{d \mid m}^{d<m} \frac{d}{m} l_{p, d} \cos \left(\frac{m}{d} \theta_{p, d}\right),
$$

where $\cos \left(\theta_{p, m}\right)=T_{p, m} / l_{p, m}, \quad l_{p, m}=\left\lceil\left|T_{p, m}\right|\right\rceil$, and $\lceil\cdot\rceil$ denotes the ceiling function. Numerically, in the range of $m$ and $p$ we explored, the occurrence of pairs $(p, m)$ requiring $l$ copies decays strongly with $l$. A value of $l=3$ is already very rare [the first occurrence was at $(p, m)=(3,1710)]$. With a fixed cutoff $m<M$ and $p<P$ it is of course always possible to find solutions of (14) in that range. This prescription then gives an identical oscillating part of the density of states as (2) up to the cutoff, and differences thereafter. Figure 2 shows the fluctuating part of the spectrum of a truncated set of butterfly graphs constructed in this manner which can be compared to Fig. 1 involving the primes. The difference in the damped high frequency oscillations is due to the truncation and would vanish when sending the cutoff to infinity. None of the partial graphs $(p, m)$ used for Fig. 2 needed copies to be taken into account.

Although in principle the $l_{p, m}$ could be unbounded, this only adds additional graphs to an already infinitely large set. Nevertheless, to obtain better control over the numbers of copies we could also construct a different set of butterfly graphs by setting $l_{p, m}=m$. An alternative approach only uses bonds of length $\ln (p)$ for each $p$. Both are detailed in the Supplemental Material [10].

Quantum graphs and the Riemann zeros.-We then have several constructions for each prime whereby infinite sets of graphs (with two bonds each) together match the oscillating contribution to the density of states that the prime contributes for the zeros of the Riemann zeta function. Combining the sets for all primes then leads to a swarm of butterfly graphs which (like the primes) pick out the Riemann zeros.

The constructions offer an explanation for the puzzling properties that a Riemann quantum chaotic system should possess - namely that the orbits should have lengths $\ln (p)$ for primes $p$ and their repetitions should all have a Maslov phase of $\pi$. The quantum graphs show that the Maslov phase of $\pi$ can actually derive from different orbits of the same length working together.

The possibility of the phase deriving from many orbits has previously been hypothesized in [21]. There Andreev reflection automatically provides the dominant periodic orbits and their odd repetitions with a Maslov phase of $\pi$ while the even repetitions would have a phase of 0 and the opposite sign. However, it was noted that including orbits of length $2^{l} \ln (p)$ for all $l \in \mathbb{N}$ could in principle compensate for the even repetitions. An alternative explanation for the overall minus sign in (2) is that the zeros correspond to an absorption spectrum [22] although this removes the necessity for the $t_{n}$ to be real.

Each butterfly graph has a simple periodic pair of wave numbers unconnected to the Riemann zeros. Combining a set of uncoupled butterfly graphs provides a composite object whose spectra is a superposition of the individual periodic spectra and whose wave functions are localized on the corresponding butterfly. The mean part of the swarm diverges (although it can be subtracted in a controlled way) and the spectra of their wave numbers become infinitely dense. Nonetheless correlations between these spectra mean the butterflies conspire to beat their wings together constructively at the Riemann zeros.

Along with the divergence of the mean density of states, a further problem is simply that the Weyl asymptotics for quantum graphs $\bar{d}(k)=L_{\mathrm{tot}} / \pi$ is independent of $k$, unlike for the Riemann zeta zeros in (1). The $H=x p$ operator, and its 'square', have also been considered on finite quantum graphs [23], with the result that the mean part of the density of states likewise cannot possibly match (1). Intriguingly however, infinite quantum graphs can be constructed with a logarithmic $k$-dependence like (1) by adding bonds of decreasing length [24].

Here, for simplicity of the constructions, we only focused on separated graphs with bond lengths given by the primes $\ln (p)$. The fact that we constructed several different systems which match (2) suggests that there are many more ways to achieve this. For example, connected orbits of other lengths could also exist as long as their contribution cancels in the end. In particular to avoid the infinite families of graphs and to reduce the divergence in the total bond length, one could imagine connecting the graphs and reusing the bonds so they contribute to many different periodic orbits.

A simple starting point could be to connect directed bonds of length $\ln \left(\frac{n+1}{n}\right)$ for $n \in \mathbb{N}_{+}$at a single vertex 
(essentially a star graph). This automatically gives infinitely many orbits of each length $\ln (p)$ by following all the bonds between $n$ and $n p-1$, along with many other lengths which of course would need to cancel. Such bond lengths are similar to those which arise from considering the full smooth part of the Riemann zeros [25]. With an identity scattering matrix at the vertex, the system would also be almost identical to the uncoupled one in [24] rescaled to provide the leading term in (1).

Returning to the butterfly graphs considered here, without the same mean part, the resulting composite spectra cannot prove the reality of $t_{n}$, but instead we propose that targeting the oscillating part of the density of states while trying to reduce the mean part could offer a possible route to finding a quantum system that exactly mimics the Riemann zeros.

This work is partly supported by the DFG (within FOR 1483). We thank Gregory Berkolaiko, Sebastian Egger, and Jonathan Keating for useful discussions and critical comments.

*jack.kuipers@ur.de quirin.hummel@ur.de

[1] D. Schumayer and D. A. W. Hutchinson, Rev. Mod. Phys. 83, 307 (2011).

[2] Y. V. Fyodorov, G. A. Hiary, and J. P. Keating, Phys. Rev. Lett. 108, 170601 (2012).

[3] Y. V. Fyodorov and J. P. Keating, Phil. Trans. R. Soc. A 372, 20120503 (2014).

[4] H. L. Montgomery, in Proceedings of the Symposium in Pure Mathematics, St. Louis, Missouri, 1972 (American Mathematical Society, Providence, 1973), p. 181.
[5] A. M. Odlyzko (unpublished).

[6] J. P. Keating and N. C. Snaith, Commun. Math. Phys. 214, 57 (2000).

[7] F. Haake, Quantum Signatures of Chaos, Springer Series in Synergetics (Springer, Berlin, 2010), 3rd ed..

[8] M. V. Berry and J.P. Keating, SIAM Rev. 41, 236 (1999).

[9] M. V. Berry, Proc. R. Soc. A 400, 229 (1985).

[10] See Supplemental Material at http://link.aps.org/ supplemental/10.1103/PhysRevLett.112.070406 for the density of zeros in the complex plane, the construction of a set of butterfly graphs with linearly growing number of copies $l_{p, m}=m$, and the construction of a set of butterfly graphs with identical bond lengths $\ln (p)$ for each prime $p$.

[11] M. C. Gutzwiller, J. Math. Phys. (N.Y.) 12, 343 (1971).

[12] G. Sierra and P. K. Townsend, Phys. Rev. Lett. 101, 110201 (2008).

[13] M. Srednicki, J. Phys. A 44, 305202 (2011).

[14] G. Sierra and J. Rodríguez-Laguna, Phys. Rev. Lett. 106, 200201 (2011).

[15] M. V. Berry and J. P. Keating, J. Phys. A 44, 285203 (2011).

[16] G. Sierra, J. Phys. A 45, 055209 (2012).

[17] G. Berkolaiko and P. Kuchment, Introduction to Quantum Graphs, Mathematical Surveys and Monographs, Vol. 186 (American Mathematical Society, Providence, 2013).

[18] P. Kuchment and O. Post, Commun. Math. Phys. 275, 805 (2007).

[19] T. Kottos and U. Smilansky, Phys. Rev. Lett. 79, 4794 (1997).

[20] S. Gnutzmann and U. Smilansky, Adv. Phys. 55, 527 (2006).

[21] M. Srednicki, Phys. Rev. Lett. 107, 100201 (2011).

[22] A. Connes, Selecta mathematica Sovietica 5, 29 (1999).

[23] S. Endres and F. Steiner, J. Phys. A 43, 095204 (2010).

[24] S. Egger né Endres and F. Steiner, J. Phys. A 44, 185202 (2011).

[25] S. Egger né Endres, Ph. D. thesis, Universität Ulm, 2011. 